\title{
CONCIENCIA Y AUTOCONCIENCIA EN KANT
}

PEDRO STEPANENKo

INSTTTUTO DE INVESTIGACIONES FILOSÓFICAS

UNIVERSIDAD NACIONAL AUTÓNOMA DE MÉXICO

El principal objetivo de este artículo es mostrar la manera como Kant justifica la tesis de origen cartesiano que sostiene que la conciencia es o presupone la autoconciencia, así como señalar qué significado debemos darle al término "autoconciencia" para evitar una regresión al infinito y para hacerla compatible con dos grandes pretensiones de la teoría kantiana del conocimiento. En la primera parte de este trabajo expongo la justificación kantiana de esa tesis cartesiana y lo hago a partir de una característica esencial de la conciencia para Kant, a saber: que la conciencia nunca es conciencia de representaciones aisladas unas de las otras. En esta misma parte señalo un absurdo en el cual puede caer la tesis cartesiana, desde la perspectiva kantiana, si se entiende por "autoconciencia" la conciencia de nuestras acciones mentales en tanto eventos que nos autoadjudicamos. Propongo, asimismo, lo que debe entenderse por "autoconciencia" para evitar ese absurdo. En la segunda parte del trabajo lo que busco es, por un lado, hacer ver cómo las ideas expuestas en la primera parte juegan un papel decisivo en la teoría kantiana del conocimiento y contribuyen a solucionar lo que me parece una fuerte objeción en su contra. Finalmente, esto me permite precisar un poco más la relación entre conciencia y autoconciencia en Kant.

\section{I}

Una de las principales propuestas de la filosofía teórica de Kant creo que debemos verla en su caracterización de la conciencia como un proceso de asimilación de información a través de una estructura conceptual. Kant se aparta de la concepción del conocimiento con esta caracterización, puesta en boga por Descartes, conforme a la cual el conocimiento es o debe ser un proceso de composición a partir de representaciones o "ideas simples". ${ }^{1} \mathrm{Ya}$ sea que las relacionemos mediante axiomas que nos permitan "derivar" otras

1 Véase Descartes, Oeuvres, ed. AT, X, pp. 417-428, 440. 
ideas, como lo indicaría el propio proyecto cartesiano, o que las asociemos de acuerdo con el orden en que la experiencia las presenta, como pensaban los empiristas británicos, lo cierto es que esta concepción presupone que tenemos conciencia de representaciones aisladas, independientes de las demás por su simplicidad y que a partir de ello construimos el resto de nuestras representaciones. En especial, Kant se opone a este empirismo que acepta que podamos tener conciencia de sensaciones separadas unas de las otras.

Quizá el pasaje en el cual pueda verse más claramente este rechazo sea el comienzo de la deducción trascendental en la primera edición de la Crttica de la razón pura (A 99). En él, Kant comienza afirmando que toda intuición, es decir, la instancia más elemental de aplicación de conceptos, contiene ya una pluralidad de representaciones que es necesario recorrer y, por lo tanto, sintetizar para tener conciencia de esa pluralidad en tanto pluralidad. Sin esa sintesis, la pluralidad no sería reconocida como tal, pues - afirma Kant- "toda representación en tanto contenida en un instante, no puede ser más que unidad absoluta" (A 99). Con esta última afirmación Kant parece estar aceptando la existencia de representaciones simples; lo que estaría negando, en todo caso,-sería la posibilidad de tener conciencia de ellas sin llevar a cabo un proceso de síntesis. En efecto, su argumento puede reconstruirse como una discusión con los empiristas, en la cual les concede que haciendo abstración de nuestra actividad pensante, los elementos últimos sobre los cuales versa la conciencia no pueden ser más que representaciones simples y aisladas unas de las otras, pero les.señala, al mismo tiempo, que no podríamos ser conscientes de esas representaciones sin presuponer nuestra actividad sintética, lo cual equivale - para Kanta presuponer el uso de conceptos, una de las condiciones indispensables de todo conocimiento posible. Así pues, Kant no niega la existencia de representaciones simples, aisladas; sólo afirma que separadas unas de las otras no forman parte de la conciencia y, por lo tanto, que no es posible sacar conocimiento alguno a partir de ellas. ${ }^{2}$ De hecho, en una carta dirigida a Marcus Herz, observa que los datos de los sentidos, independientemente de las condiciones del conocimiento, podrían tener "influencia sobre mis deseos y sentimientos sin que yo tenga conocimiento de ellos" (A.A., XI, p. 52). Puede haber, pues, representaciones aisladas, sólo que para mí, en tanto "ser cognioscente", no son nada. Para que una representación forme parte de la conciencia, necesita ser aprehendida mediante el uso de algún concepto, "por muy imperfecto u oscuro que éste sea" (A 106).

2 Yo creo que esto pone en cuestión lo que Strawson afirma en The Bounds of Sense acerca de la teoría kantiana de la síntesis: que presupone la creencia en impresiones inconexas (Methuell, Londres, 1966, p. 32). 
En la sección a la cual pertenece el pasaje de la deducción antes mencionado pueden destacarse dos justificaciones de esta idea, según la cual no es posible tener conciencia de intuiciones (a las cuales pertenecen las sensaciones) sin hacer uso de conceptos. La primera y más elemental podría exponerse de la siguiente manera: para tener conciencia de la pluralidad que constituye una intuición tengo, al menos, que distribuir temporalmente las representaciones contenidas, pero esta serie temporal que debo formar sería imposible si no pudiera identificar, distinguir o reidentificar cada una de las representaciones de la serie. Para saber de una pluralidad como tal debo recordar las representaciones que he recorrido, pero debo hacerlo de tal manera que no las confunda con nuevas representaciones, para lo cual es necesario subsumir cada representación bajo algún concepto. Y este acto de subsunción implica relacionar la representación subsumida con todas las demás representaciones que puedan caer bajo el mismo concepto. Para formar la serie temporal de representaciones debo, por decirlo de alguna manera, sacar cada representación del flujo del tiempo, por paradójico que suene. Debo situarme mediante el uso de conceptos fuera del flujo del tiempo, para poder ubicar una serie de representaciones en el tiempo.

Esta explicación, aunque sugerida por algunos pasajes, en realidad no responde a la peculiar perspectiva kantiana. La justificación que en verdad le interesa a Kant depende de la particular manera en que caracteriza un concepto, a saber: como una representación general que sirve de regla, conforme a la cual enlazamos una pluralidad de representaciones particulares. ${ }^{3}$ Los conceptos para Kant deben servir como una guía que nos permite ir formando series con una pluralidad de representaciones, por sí mismas caóticas o indiferenciadas. Es más, son aquello que ordena y coordina nuestra actividad pensante, pero no como una reflexión sobre los datos de los sentidos, sino precisamente como aquello que hace posible integrar esos datos a la conciencia. Es esta caracterización la que explica por qué Kant llama al concepto "unidad de la síntesis", con lo cual no se refiere al resultado de la síntesis, sino a la forma de la misma o a la regla de acuerdo con la cual se desenvuelve. También es esta manera de concebir los conceptos lo que justifica inmediatamante negar la conciencia de intuiciones sin hacer uso de conceptos, pues éstos son los que determinan la forma en que recorremos la pluralidad que constituye toda intuición. Recorrer una pluralidad significa siempre recorrerla de una manera específica y esta manera es lo que expresamos mediante un concepto. Sería imposible recorrer una pluralidad sin tener idea de cómo la recorremos, sería incluso imposible distinguir unas representaciones de otras, pues no sabríamos ni siquiera bajo qué aspecto 
son distintas; estaríamos siempre ante un conjunto caótico de representaciones del cual no podríamos ni siquiera saber que es el mismo que cuando. empezamos a recorrerlo.

Sin conciencia de que aquello que pensamos es precisamente lo mismo que pensábamos un momento antes, sería inútil toda reproducción en la serie de las representaciones. Pues, en el estado actual habría una nueva representación, que de ninguna manera pertenecería al acto por el cual debió ser producida gradualmente; $y$ la pluralidad de la misma jamás constituiría un todo, porque haría falta la unidad que sólo la conciencia puede proporcionar. Si al contar olvido que las unidades que ahora tengo ante mí han sido añadidas sucesivamemte por mí una a la otra, entonces no reconoceré la producción del conjunto por esa adición sucesiva de una unidad a la otra y, consecuentemente, tampoco el número; pues este concepto consiste solamente en la conciencia de la unidad de la síntesis. (A 103) ${ }^{4}$

No aceptar que haya conciencia de representaciones aisladas, por las razones que he tratado de exponer anteriormente, le permite a Kant adoptar la tesis cartesiana, según la cual la conciencia es o presupone siempre la autoconciencia. ${ }^{5}$ Pero me parece que Kant puede justificar esta tesis de una manera más sencilla y convincente que la del propio Descartes. Esta justificación creo que podría reducirse a la siguiente observación: si para tener conciencia de una representación es necesario enlazarla con otras representaciones, entonces es necesario también tener conciencia de ese enlace; la conciencia de cualquier representación intuitiva presupone, pues, la autoconciencia o, como Kant la llama, la apercepción. En donde Kant expresa más claramente esta tesis es en una serie de reflexiones ("los manuscritos de Duisburg") que escribió hacia 1775, en el periodo decisivo de gestación de la filosofía crítica. En una de estas reflexiones Kant afirma:

Percibimos algo sólo porque somos conscientes de nuestra aprehensión... (R 4681; A.A., XVII, 667)

y en otra:

Somos conscientes de nosotros mismos, de nuestras acciones y de los fenómenos [Erscheinungen] en la medida en que tenemos conciencia de su aprehensión, ya sea a través de coordinarlos entre sí o de aprehender una sensación por otra. (R 4679; A.A., XVII, 662)

4 Todas las traducciones de las citas de Kant son mias.

5 Véase Descartes, Oeuvres, ed. AT, VII, p. 246/Meditaciones metafisicas, trad. Vidal Peña, Alfaguara, Madrid, 1977, p. 198. 
En la Crítica de la razón pura, sin embargo, no es posible encontrar un pasaje que exprese con esta nitidez la relación de presuposición entre la conciencia de representaciones intuitivas y la conciencia de la acción de enlazarlas. Esto se debe, fundamentalmente, a mi modo de ver el asunto, a dos razones: una de ellas es que esta tesis aparece en la Crítica entremezclada con otras tesis acerca de la relación entre la autoconciencia y la conciencia de una pluralidad, en donde por "autoconciencia" se entiende básicamente la conciencia de nuestra identidad. ${ }^{6}$ Pero la principal razón por la cual en la Crítica no se encuentran pasajes como aquellos de los manuscritos de Duisburg es porque en ellos parecen confundirse la conciencia empírica de nosotros mismos con la autoconciencia trascendental, diferencia en la que Kant insiste permanentemente en la Crítica y que en los manuscritos apenas está esbozando. La autoconciencia empírica es la conciencia que tengo de mí mismo en tanto fenómeno del sentido interno. Para este tipo de conciencia es necesario que me autoadjudique ciertas experiencias o acciones, que tengo que ubicar en el tiempo. La autoconciencia trascendental, en cambio, es sólo conciencia de la regla o de las reglas, conforme a las cuales determino los fenómenos en el tiempo, incluyéndome a mí mismo. Si no se distinguen estos dos tipos de autoconciencia, Kant estaría exigiendo algo muy extraño al sostener que, para ser consciente de una serie de representaciones en el tiempo, se requiere tener conciencia de la propia síntesis. $\mathrm{Si}$ esta última fuera una autoconciencia empírica, entonces habría que enlazar, a su vez, la representación de esta acción de sintetizar con otras representaciones de otras acciones, para ser conscientes de la misma, pero al enlazarla tendríamos, de nuevo, que tener conciencia de este último enlace, desencadenándose de esta manera una regresión al infinito, parecida a la que le objetaban a Descartes. ${ }^{7}$ En una reflexión posterior a las dos ediciones de la Crítica de la razón pura, en la cual se plantea el problema de si pensar es una experiencia, Kant advierte precisamente este peligro de esa confusión.

La conciencia, cuando llevo a cabo una experiencia, es una representación de mi existencia en tanto determinada empíricamente, es decir, en el tiempo. Si esta conciencia fuera a su vez empírica, entonces la misma determinación [acción de determinar] del tiempo tendría a su vez que ser representada, como contenida bajo las condiciones de la determinación temporal de mi estado. Tendría, pues,

${ }^{6}$ Cfr. Hernrich D., Identität und Objektivität. Eine Untersuchung über Kants transzendental Deduktion, Heildelberg, C., Winter, 1976, pp. 54-107 / "Die Identität des Selbstbewußtsein in der transzendentalen Deduktion" en Oberer, H. y Seel, G., Kant: Analysen -ProblemeKritik, Würzburg, Köningshausen y Neumann, 1988, pp. 39-70.

7 AT, VII, p. 173 y p. $413 / \mathrm{MM}$, trad. Vidal Peña, p. 141 y p. 315. Véase también mi artículo "El concepto de conciencia cartesiano y la objeción del regreso al infinito de pensamientos" en Diánoia, no. 39, 1993. 
que ser pensado otro tiempo bajo el cual (no en el cual) el tiempo, que constituye la condición formal de mi experiencia interna, estuviera contenido. Habría, pues, un tiempo en el cual y con el cual al par un tiempo dado correría, lo cual es absurdo. Pero la conciencia de tener una experiencia o, en general, de pensar es una conciencia trascendental, no una experiencia. (A.A., XVIII, 319; el subrayado es mío.)

Al tener una experiencia, me es dada una pluralidad que sintetizo en una serie temporal. Tengo conciencia de esa pluralidad en tanto que las distintas representaciones están integradas en esa serie. Esa serie puede referirse a la secuencia que constituyen mis estados mentales. Pero de la acción de sintetizar no es posible decir que tengo una experiencia al mismo tiempo que formo la otra serie; eso equivaldría a decir que al formar una serie temporal de representaciones intuitivas, compongo también una serie con las representaciones de las acciones de sintetizar, y una vez aceptado esto, habría que agregar que también construyo otra serie con las acciones que me permiten formar esta última serie. Mucho menos podría decirse que la primera serie presupone la segunda. Esa conciencia de la síntesis tiene, pues, que prescindir del aspecto temporal de la misma y enfocar exclusivamente la regla 0 el concepto que la determina, por eso Kant afirma, en esta reflexión, que la conciencia de pensar no es una experiencia, no es una conciencia empírica.

En la Crítica de la razón pura Kant no cae en el absurdo anteriormente expuesto, porque entiende por "autoconciencia trascendental" la conciencia de la unidad de la síntesis, no la conciencia de la acción en tanto que se lleva a cabo en un determinado momento. A esto se debe, entre otras cosas, la extraña expresión de "unidad sintética de la apercepción". Con ella Kant pone énfasis en que la autoconciencia trascendental es conciencia de la unidad que hace posible la síntesis, que es un saber del concepto o conceptos que hacen posible el enlace de representaciones, no un saber acerca de nuestra existencia en el tiempo. Así pues, la relación de presuposición que Kant sostiene entre la conciencia de una pluralidad y la autoconciencia hay que expresarla de la siguiente manera: no hay conciencia de representaciones particulares sin conciencia de la unidad que hace posible enlazarlas en una representación compleja.

\section{II}

Hasta aquí he abordado una de las principales ideas de Kant con respecto a la relación entre conceptos y representaciones intuitivas, pero lo he intentado hacer ignorando las dos grandes pretensiones que la enmarcan en la teoría kantiana del conocimiento. Una de ellas sostiene que debe haber un solo conjunto de categorías o conceptos elementales, bajo el cual se hallan 
todas las representaciones de la experiencia. ${ }^{8}$ La otra, que ese conjunto de categorías o esquema categorial es to que nos permite referir representaciones a objetos y de esta manera distinguir representaciones objetivas de representaciones subjetivas. ${ }^{9} \mathrm{Y}$ es fundamental reubicarla para comprender la posición de Kant, ya que esta idea, según la cual no es posible aprehender representaciones de la experiencia sin conceptos que nos permitan relacionarlas con otras, en realidad forma parte del argumento de la deducción trascendental en favor de la necesidad de un solo esquema categorial bajo el cual pensamos. Modificando un poco el asunto, podría decirse incluso que es el primer paso de ese argumento. El segundo paso consistiría en hacer notar que la conciencia de nuestra identidad es también necesaria para concebir una pluralidad de representaciones, pues si no supiéramos que somos los mismos a cada momento que recibimos una representación, entonces no podríamos considerarla distinta de otras que hemos aprehendido previamente. Y en el último paso habría que señalar que sólo un conjunto de reglas o conceptos puede tomarse como aquello que permanece idéntico en nosotros, ya que, en cuanto a las representaciones que asimilamos, siempre hay variaciones. Lo que constituye, pues, nuestra identidad en tanto sujetos del conocimiento es un esquema conceptual siempre el mismo; si pudiéramos cambiar las reglas más elementales, gracias a las cuales relacionamos representaciones, nos quedaríamos sin criterio alguno para decir que somos los mismos y la pluralidad de representaciones que constituye la experiencia de un sujeto quedaría a tal punto desarticulada que no podría afirmarse que se tiene conciencia de la misma. Si en una partida de ajedrez fuera posible cambiar las reglas del juego, no podríamos decir que seguimos jugando la misma partida. Éste es, a grandes rasgos, el argumento por el cual Kant sostiene que debe haber un único conjunto de categorías; y si es único, se aplica necesariamente, porque, de acuerdo con el primer paso, no hay experiencia sin conceptos. Todas las representaciones de la experiencia tienen, pues, que hallarse bajo un mismo esquema categorial. La unidad sintética de la apercepción creo que debe entenderse precisamente como la unidad de esos conceptos elementales que determinan nuestra identidad y bajo la cual deben hallarse todas las representaciones de la experiencia.

En cuanto a la otra gran pretensión de la teoría kantiana del conocimiento: que ese esquema categorial único es el que nos permite referir representaciones a objetos, tiene una historia larga y complicada que no podré narrar

8 Con respecto a la equivalencia entre sostener la necesidad de un esquema categorial y afirmar que hay un único esquema categorial, véase Körner, S., "The Impossibility of Trascendental Deductions", en Beck, L.W. (ed.), Kant Studies Today, La Salle, 1969, pp. 230-244.

9 Cfr. Guyer, P., "The Trascendental Deduction of the Categories", en Guyer, P. (ed.), The Cambridge Companion to Kant, Cambridge University Press, Cambridge, 1992, pp. 129-133. 
aquí, pero sí me parece importante señalar que esta pretensión descansa en una convicción que Kant hereda de la filosofía wolffiano-leibniziana, a saber: que hay conceptos a priori a través de los cuales pensamos lo que es un objeto en general y que la existencia de estos conceptos es lo único que garantiza la existencia de la metafísica. La originalidad de Kant en el enfoque de estos conceptos consiste en querer justificar su objetividad apelando a la función que desempeñan en la experiencia. Esto es lo que hizo a Kant concebir estos conceptos como criterios por los cuales consideramos que a nuestras representaciones les corresponden objetos que existen con independencia de esas representaciones, en otras palabras, como aquellas relaciones entre representaciones, gracias a las cuales pensamos en objetos que les corresponden a las representaciones. En los Prolegomena Kant explica esta labor de las categorías o conceptos puros del entendimiento distinguiendo dos tipos de juicios: los juicios de percepción y los juicios de experiencia. ${ }^{10}$ En los juicios de percepción tan sólo describo las representaciones tal como me son dadas, como, por ejemplo, al decir "cuando brilla el sol y toco esta piedra, siento caliente la piedra". En los juicios de experiencia, en cambio, aplico una categoría por la cual pienso en la relación de mis representaciones como una relación entre objetos. El juicio correspondiente en este caso sería: "el sol calienta esta piedra" o "la causa del calor en esta piedra es el brillo del sol". Esta distinción entre tipos de juicios también puede expresarse mediante la distinción entre representaciones subjetivas y objetivas. La diferencia entre ellas consiste en que al pensar o tener conciencia de las primeras sólo sé de ellas en tanto representaciones, mientras que a través de las representaciones objetivas pienso en los objetos que les corresponden. Así pues, puede decirse que las categorías son aquellos criterios mediante los cuales distinguimos representaciones subjetivas de representaciones objetivas. Y estos criterios no expresan más que relaciones entre representaciones.

En la Crítica de la razón pura lo que hace Kant es identificar estas categorias, por las que pensamos en objetos correspondientes a nuestras representaciones, con aquellos conceptos elementales que dan a nuestras representaciones la coherencia que nos permite hablar de unidad e identidad de la conciencia. Lo hace a partir de una reflexión acerca de un problema clásico del conocimiento, que en sus lecciones de lógica llama "dialelo", 11 a saber: el hecho de no poder confrontar nunca nuestras representaciones con su objeto, sino sólo nuestras representaciones entre sí, y esto a pesar de aceptar que el conocimiento es adecuación de nuestras representaciones con un objeto o hecho independiente de las mismas.

10 Prolegomena, $\S \S 18-20$.

11 Immanuel Kants Logik. Ein Hanbuch zu Vorlesungen, A 70. 
¿Qué se entiende, pues, cuando se habla de un objeto que corresponde a un conocimiento y que, por lo tanto, es diferente de él? Es fácil ver que este objeto sólo puede ser pensado como algo general $=x$, puesto que no tenemos nada, más que nuestro conocimiento, que podamos colocar ante ese conocimiento como correspondiente.

Hallamos, sin embargo, que nuestro pensamiento acerca de la referencia de todo conocimiento a su objeto conlleva algo de necesidad, ya que éste [el objeto] es considerado como aquello ante lo cual nuestros conocimientos no se determinan al azar o como se quiera, sino a priori de cierta manera, porque, en tanto que deben referirse a un objeto, tienen que concordar entre sí en relación con éste necesariamente, es decir, tienen que poseer aquella unidad que constituye el concepto de un objeto.

Pero, ya que sólo tenemos que ver con la pluralidad de nuestras representaciones y aquella $x$ que les corresponde (el objeto) no es nada para nosotros, porque debe ser algo diferente de todas nuestras representaciones, está claro que la unidad que el objeto hace necesaria no puede ser más que la unidad formal de la conciencia, en la síntesis de la pluralidad de representaciones. (A 104/5)

Lo que Kant entiende aquí por la "unidad que constituye el concepto de un objeto" no es más que el conjunto de categorías que expresan las relaciones entre representaciones, gracias a las cuales pensamos en sus objetos correspondientes, de tal manera que la identificación que lleva a cabo Kant es entre este conjunto y la "unidad formal de la conciencia", es decir, los conceptos elementales que constituyen la identidad del sujeto del conocimiento. Esta tesis es muy fuerte y yo creo que forma el núcleo de la teoría del conocimiento de Kant. Muchas generaciones de filósofos han tratado de interpretarla y entre los múltiples problemas que puede generar dentro de la propia filosofía de Kant, destaca uno al que se le ha denominado "el problema de las representaciones subjetivas". ${ }^{12}$ Este problema puede formularse de la siguiente manera: ya que Kant pretende haber demostrado que toda representación tiene que hallarse bajo la unidad formal de la conciencia, es decir, que tiene que estar enlazada mediante categorías con otras representaciones, entonces identificar esta unidad con la unidad del objeto significa que toda representación es ya objetiva, por el hecho mismo de que tengamos conciencia de ella. Si las categorías son las que determinan la unidad que

12 Este problema ha sido abordado por L.W. Beck en su articulo "Did the Sage of Könisberg Have no Dreams?" (en Essays on Kant and Hume) que, a su vez, parte de una objeción que C.I. Lewis presenta en contra de la teorfa kantiana de las categorfas, expresada precisamente por esa pregunta con la que Beck titula su articulo. Conforme a Lewis (Mind and the World Order, Nueva York, 1929, p. 221) la deducción de las categorías conduce al absurdo de no reconocer la conciencia de sueños y, en general, de cualquier representación subjetiva. B. Thöle también ha tratado este problema en su libro Kant und das Problem der Gesetzmäßigkeit der Natur. 
el objeto hace necesaria y, a la vez, no es posible tener conciencia de representación alguna que no esté enlazada con otras conforme a categorías, entonces toda representación es objetiva. Las categorías como criterios para distinguir representaciones objetivas de representaciones subjetivas son, entonces, inútiles, puesto que no hay representaciones subjetivas, en el sentido de representaciones no enlazadas conforme a categorías. Podría señalarse, como lo hace Lewis White Beck, ${ }^{13}$ que las representaciones subjetivas se refieren a nosotros mismos en tanto sujetos del sentido interno, en tanto fenómenos, pero esto no resuelve el problema, ya que nosotros mismos en tanto fenómenos somos un objeto más en el mundo y lo que podemos saber de este objeto requiere igualmente de la aplicación de categorlas. Parece, pues, que Kant debió haber renunciado a la diferencia entre representaciones objetivas y subjetivas, si quería sostener la identificación entre la "unidad formal de la conciencia" y la unidad que constituye el concepto de un objeto en general. Pero renunciar a esta diferencia creo que conduce a una funesta consecuencia, a saber: renunciar a la posibilidad de corregir o cambiar la aplicación de conceptos particulares a representaciones intuitivas. En efecto, si subsumir una representación intuitiva bajo un concepto significa relacionarla con otras representaciones conforme a reglas, el único motivo que podríamos tener para sustituir un concepto por otro sería la presencia de representaciones que no pueden ser sintetizadas por el primer concepto. Es cierto, si sustituimos un concepto por otro es debido a la exigencia de integrar las representaciones aún no sintetizadas a una representación unitaria, pero esto no significa que ya las hayamos enlazado con otras, conforme a una regla.

Podría objetarse que la sustitución de un concepto por otro no nos constriñe a aceptar representaciones no sintetizadas. Podríamos pensar que las representaciones que se asimilan con el nuevo concepto, y que no sintetizaba el concepto que sustituimos, ya estaban enlazadas con otras representaciones bajo otro $u$ otros conceptos, de tal manera que la sustitución de un concepto por otro nos obligaría a sustituir varios conceptos y, por así decirlo, llevar a cabo una nueva distribución de intuiciones. Esta manera de entender la sustitución de un concepto por otro no nos exige aceptar que haya representaciones intuitivas que no se encuentren ya enlazadas por las categorías. Sin embargo, sí nos obliga a aceptar que hay un orden de representaciones $o$ un cierto tipo de relaciones entre las mismas que no depende de la aplicación de categorias, pues, de no ser así, no habría razón alguna para considerar que ciertas representaciones sintetizadas por un concepto tenemos que enlazarlas con las representaciones que caen bajo otro concepto y de tal manera que tengamos que sustituir ambos conceptos.

13 Op. cit. 
Yo creo que Kant se percató de las dificultades a las que le conducía identificar la unidad del objeto en general y la unidad de la conciencia y, por ello, entre otras razones, modificó la deducción trascendental en la segunda edición de la Crítica de la razón pura. En esta edición Kant pone mayor énfasis en que la conciencia de una pluralidad presupone pensar o tener conciencia de la unidad en la cual puede integrarse esa pluralidad, pero no necesariamente en la unidad que expresa la síntesis que ya manifiestan las representaciones. Esto le permite aceptar representaciones subjetivas sin abandonar la necesidad de pensar un orden objetivo en el cual puedan integrarse: son subjetivas en tanto que aún no están sintetizadas, pero son objetivables en tanto que pensar en ellas significa buscar la posición que deben tener en el orden objetivo que establecen las categorfas.

El pensamiento: estas representaciones dadas en la intuición me pertenecen todas juntas, quiere decir [...] lo mismo que: las enlazo en una autoconciencia 0 , al menos, pueden ser enlazadas en ella. Y si bien ese pensamiento no es todavía la conciencia de la síntesis de las representaciones, presupone la posibilidad de esta última, es decir, sólo porque puedo concebir [begreifen] la pluralidad de las mismas en una conciencia, las llamo en conjunto mis representaciones. (B 133; el subrayado es mío)

Tener conciencia de una pluralidad de representaciones, saber que me pertenecen, presupone pensarlas como sintetizables, pensar en el orden en el cual pueden adquirir una posición determinada entre otras representaciones, es decir, en la unidad objetiva de la autoconciencia, pero no necesariamente saber ya del enlace de las mismas en esa unidad.

Esta manera de condicionar la conciencia de roda pluralidad a la unidad sintética de la apercepción permite aceptar un orden de representaciones no encadenado de acuerdo con las categorías, un orden subjetivo de representaciones, el cual, sin embargo, en tanto que pensamos en él, se halla condicionado por pensar en la unidad estructurada por las categorfas. Siguiendo esta idea, podría incluso decirse que pensar una pluralidad no significa más que mover las representaciones sobre un fondo siempre idéntico, moverlas para hacerlas encuadrar en la unidad siempre idéntica de la autoconciencia, es decir, buscar la posición fija que les corresponde en el todo coherente de las representaciones. De esta manera, la conciencia no resulta ser un proceso rígido de asimilación de representaciones, sino un juego entre una estructura fija que nos permite referirlas a objetos y representaciones que pueden ocupar distintas posiciones en esa estructura. 